\title{
Diplomyelia in a patient with a clinical suspicion of autosomal recessive spastic ataxia of Charlevoix-Saguenay type (ARSACS)
}

\author{
Dorota Dziewulska \\ Department of Neurology, Medical University of Warsaw, Poland
}

\begin{abstract}
Autosomal recessive spastic ataxia of Charlevoix-Saguenay (ARSACS) is a form of cerebellar ataxia related to mutations in the SACS gene on chromosome 13912.12 encoding sacsin protein. Characteristic clinical features are ataxia, spasticity, distal muscle wasting, neuropathy, dysarthria, nystagmus, and finger or feet deformities.

The presented case concerns a 32-year-old man with clinical diagnosis of ARSACS. Magnetic resonance imaging (MRI) scans of the brain revealed cerebellar atrophy typical of the disease while neuroimaging of the C1-C3 and C6-Th12 segments showed only the thin thoracic spinal cord. The patient died suddenly and a gross examination of the spinal cord revealed extraspinal tumour at the C4-C5 levels, which turned out to be an additional spinal cord. Microscopic examination showed an extensive ischemic necrosis involving C6-Th5 segments of the proper spinal cord, and disturbed intrinsic structure containing many pathological vessels of the extra spinal cord.

Lack of visualization of C4-C5 spinal cord segments on MRI scans made diagnosis of diplomyelia in vivo impossible. However, diplomyelia does not exclude coexistence of ARSACS because of the occurrence of such clinical symptoms as dysarthria or nystagmus which cannot be explained by the presence of the spinal cord defect. The possibility of congenital malformations of the spinal cord in adults should be remembered as their early identification and surgical correction can improve neurological symptoms.
\end{abstract}

Key words: ARSACS, cerebellar ataxia, Charlevoix-Saguenay, diplomyelia.

\section{Introduction}

Autosomal recessive spastic ataxia of CharlevoixSaguenay (ARSACS) is a distinct form of early-onset cerebellar ataxia with progressive degeneration of cerebellum and spinal cord. The disorder was first described in the 1970s among inhabitants of the Charlevoix-Saguenay region in north-eastern Quebec in Canada [3]. ARSACS is related to mutations in the SACS gene on chromosome 13q12.12 encoding a large multidomain protein sacsin. Sacsin is involved in chaperon-mediated protein folding and regulation of the effects of other ataxia-associated proteins [11]. Although the molecular mechanism underlying ARSACS remains unknown, probably the disease is associated with a loss of sacsin function. The protein has a predominantly cytoplasmic distribution with a mitochondrial component and is most highly expressed in large neurons, particularly within brain motor systems, including cerebellar Purkinje 
cells. It is also present in fibroblasts, skeletal muscles and axons [11].

More than 70 different mutations in the sacsin gene have been identified worldwide [2] causing phenotypic variability of ARSACS even within family members harbouring the same mutation. However, characteristic clinical features of the disease are cerebellar ataxia, spasticity, distal muscle wasting, axonal polyneuropathy, dysarthria, nystagmus, and finger or/and foot deformities. The presented case concerns a 32-year-old man with a clinical suspicion of ARSACS and unexpected finding in the autopsy examination - a spinal cord duplication.

\section{Case report}

A 32-year-old patient with diagnosed motor neuropathy at the age of 16 years and negative family history, was admitted to the hospital for the re-evaluation of the neurological condition. The first symptoms of the disease in the form of feet drop occurred at the age of 14 years, and then gradually intensified spastic lower limb (LL) paresis, and then upper limb (UL) disability was observed. In the neurological examination, dysarthric speech, horizontal nystagmus, advanced distal muscular atrophy in limbs (more severe in legs than hands), feet drop, spastic muscle tone in LLs, excessive deep tendon reflexes, superficial and deep sensory disturbances in LLs, and neurogenic urinary bladder were described.

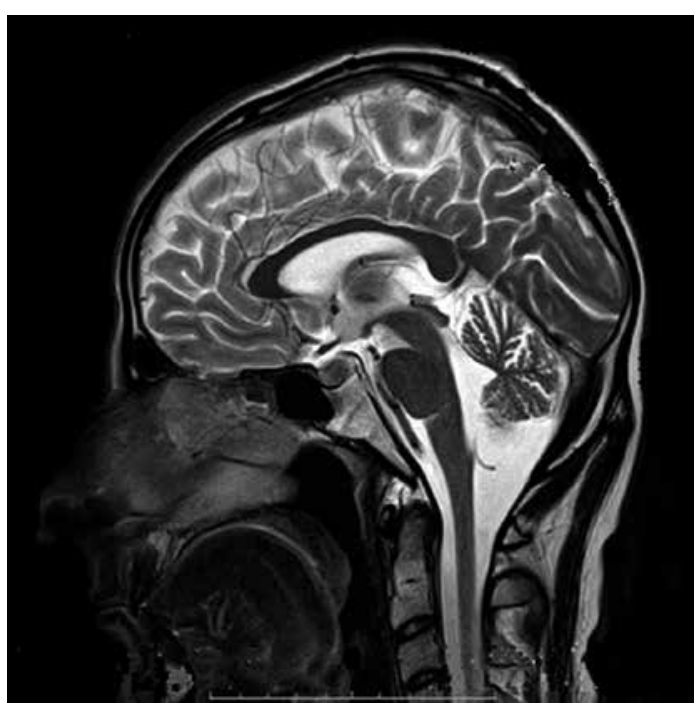

Fig. 1. Brain MRI scan demonstrates mild atrophy of the cerebellar vermis.
Electroneurogram (ENG) revealed axonal damage to motor fibres, and electromyography (EMG) exhibited neurogenic record in LL muscles but normal in UL muscles. Brain magnetic resonance imaging (MRI) also demonstrating the upper part of the cervical spinal cord (up level of (3) showed atrophy of the cerebellum, especially of the upper vermis (Fig. 1), but MRI of the thoracic spinal cord also including the lower cervical segments (C6-C7) was normal except for narrowing of the thoracic spinal cord. Routine biochemical tests and psychologic examination were normal. Although cerebellar ataxia was not observed in our patient, based on the clinical picture (childhood onset spastic distal paraparesis, dysarthria, nystagmus, sensory disorders) (Fig. 2) and results of additional tests (upper cerebellar vernix atrophy in MRI, axonal polyneuropathy in ENG), ARSACS was clinically diagnosed.

The patient died suddenly at home one year after the last hospitalization. The death followed sudden dyspnoea, anarthria, loss of sphincter control, and coma. Autopsy showed pulmonary oedema. General brain examination revealed cerebellar atrophy and cerebral oedema with cerebellar tonsil invagination. In microscopic examination of the brain, tissue oedema and only a minimal loss of Purkinje cells were noted. Spinal cord examination showed a crescent extraspinal mass (tumour) $30 \times 15 \times 5 \mathrm{~mm}$ at C4-C5 segments (Fig. 3A-D). The pathological mass adhered to the right side of the spinal cord and was

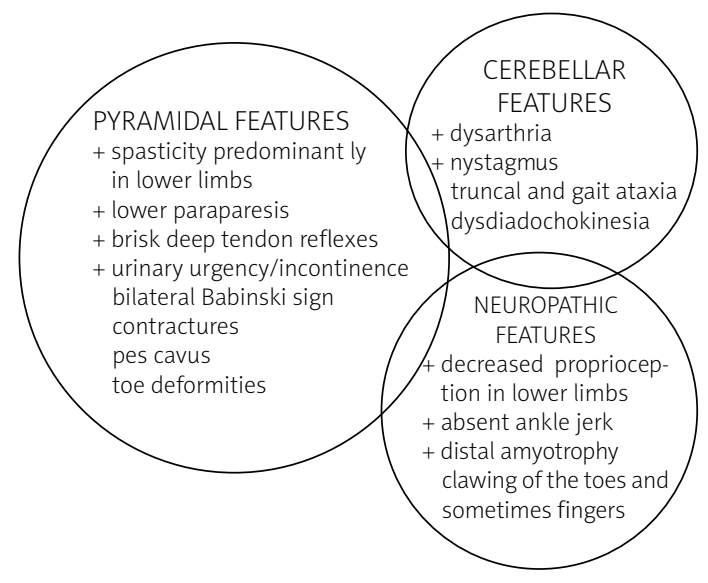

Fig. 2. The spectrum of ARSACS symptoms. Symptoms marked with + were present in our patient (adapted from [1]). 
A
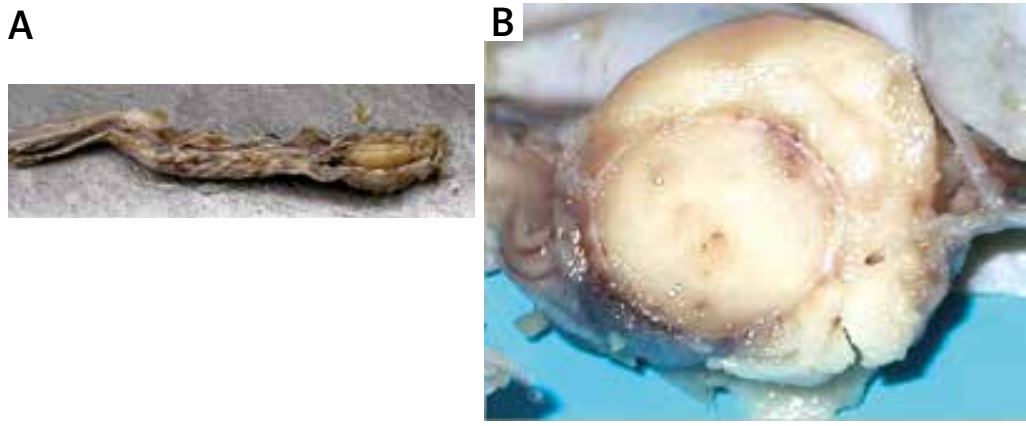

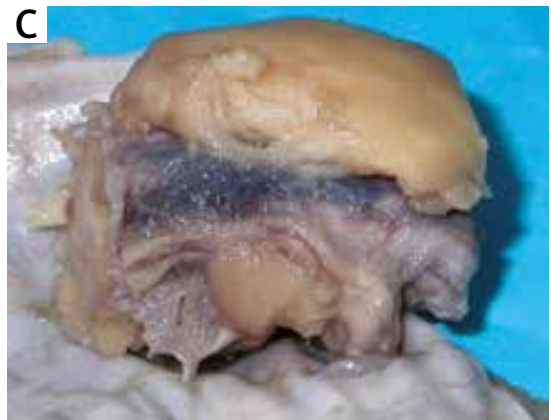

Fig. 3. Spinal cord autopsy. A) Cervico-thoracic fragment of the spinal cord with visible intradural extraspinal tumour at the C4-C5 level. B) Cross section showing the crescent tumour (additional spinal cord) surrounding the proper spinal cord. C) Longitudinal section showing the additional spinal cord and vessels between it and the proper spinal cord.

not fused with either the dura matter or the spinal cord. After removing the tumour, abnormal vessels on the posterior surface of the spinal cord appeared. At the levels of C4-C6, the anterior spinal artery was doubled (Fig. 4C). Below the tumour, the C6-Th5 spinal cord segments showed thinning and flattening with a significant reduction in tissue cohesion. In the thoracic and lumbar segments, anterior spinal roots were thinner than the posterior ones.

On a microscopic examination, C4-C5 segments of the proper spinal cord showed notable asymmetry, disturbed intrinsic structure with clusters of
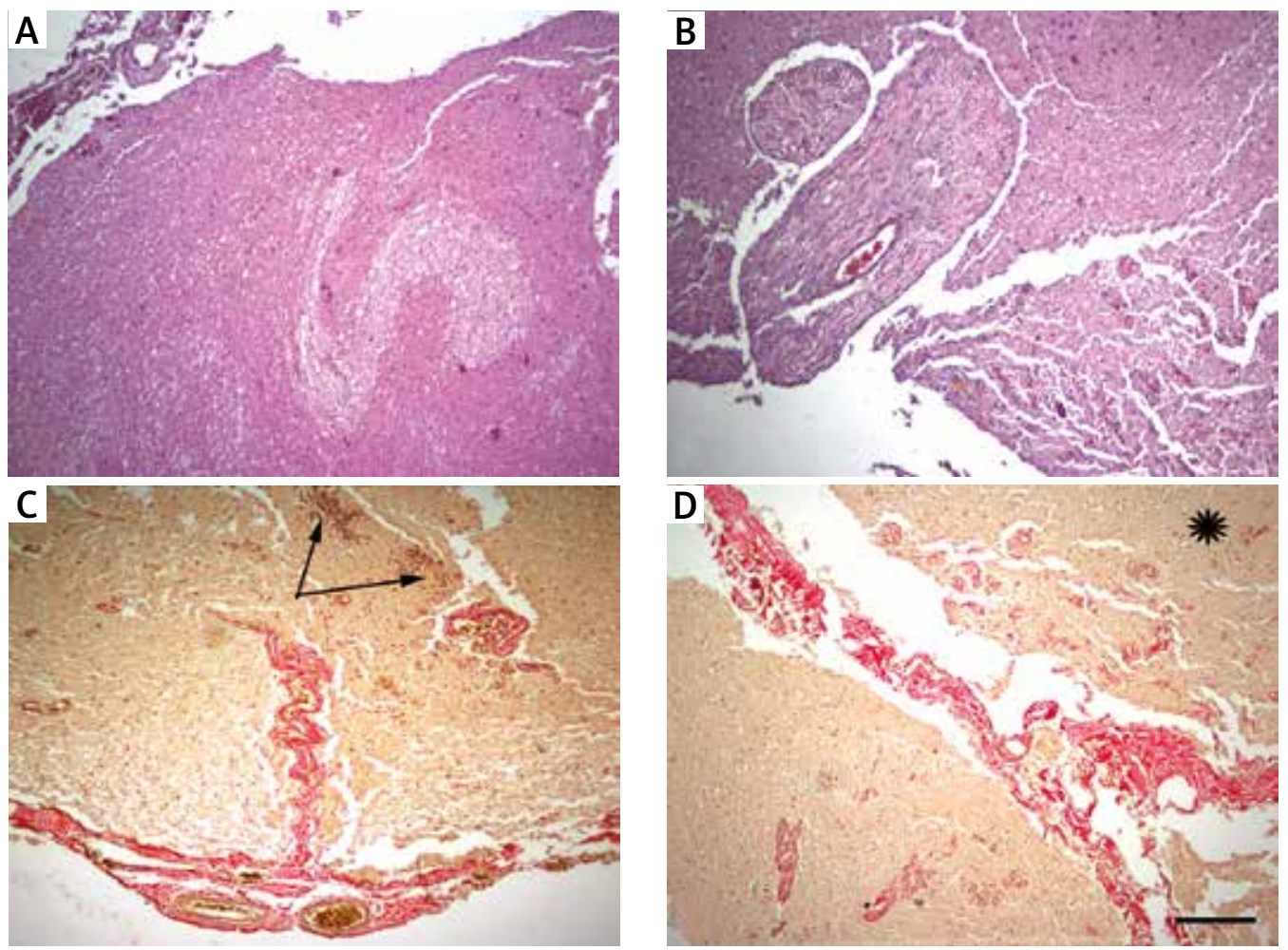

Fig. 4. Microscopical examination of the spinal cords. A) Area of necrosis and spongiosis in the additional spinal cord; HE. B) Single spinal roots of the additional spinal cord; HE. C) C5 segment of the proper spinal cord - visible doubled anterior spinal artery and clusters of ependymal cells (arrows); van Gieson. D) Connective tissue with vessels separating the proper (asterisk) and additional spinal cord. Numerous pathological vessels are visible in the adjacent areas of both spinal cords; van Gieson, scale bar - $100 \mu \mathrm{m}$. 
ependymal cells in the grey matter, and pathological vessels in the area adjacent to the additional spinal cord. An extensive ischemic necrosis involving C6-Th5 segments was found there.

On a microscopic examination, the macroscopically visible tumour turned out to be an extra spinal cord surrounded by leptomeninges. The additional spinal cord also had disturbed the internal structure with clusters of ependymal cells, single neurons, irregular foci of spongiosis and tissue oedema (Fig. 4A). Only a few spinal roots emerged from the extra spinal cord (Fig. 4B). In contrast to the proper spinal cord, many pathological vessels were observed in the additional spinal cord throughout the entire neuropil, not just in areas adjacent to the proper spinal cord (Fig. 4C,D).

\section{Discussion}

In the presented case, unexpected malformation of the spinal cord was found during autopsy. The direct cause of the patient's death was probably respiratory failure due to among others intercostal muscles paralysis in the course of acute ischemic necrosis of the thoracic spinal cord. But the presence of diplomyelia does not exclude the coexistence of ARSACS, because some symptoms observed in our patient such as nystagmus, and dysarthria cannot be explained solely by the presence of spinal cord malformation. There is limited autopsy material available from cases of ARSACS. The pathologic findings in a young man published in 1991 [4] showed focal rarefaction of the Purkinje cells in the cerebellum, demyelination along the corticospinal tracts in the medulla and spinal cord, and small corticospinal tracts in the pons with normal pontine nuclei. On thalamus and cerebellar cortex swollen neurons with dense lipofuscin-like granules within their lysosomes were also observed. In our case, except loss of Purkinje cells, the above-mentioned non-specific brain changes were not found.

ARSACS belongs to a complex group of at least 20 autosomal recessive cerebellar ataxias with different clinical phenotypes varying from predominant cerebellar syndromes to sensorimotor neuropathy, seizures, involuntary movements, cognitive dysfunction, skeletal and cutaneous disorders, and even cases without ataxia [1]. In ARSACS, not ataxia but spasticity is considered as a core clinical feature [2] (Fig. 2), and a predominant truncal and gait ataxia was seen only in inhabitants of the Charlevoix-Saguenay region [1]. In non-Quebec patients, especially in late-onset variants of the disease, ARSACS may present with isolated spasticity, Charcot-Marie-Tooth-like phenotype, adult not childhood onset ataxia [12]. In our case, cerebellar ataxia was not observed, but in a case with spastic lower paraparesis it is problematic to distinguish whether gait disturbances are due to muscle weakness or cerebellar-related discoordination. However, in our patient asymptomatic cerebellar involvement (vermis atrophy and loss of Purkinje cells) was found. In such doubtful cases, the final confirmation of ARSACS would be a genetic test showing the presence of a mutation in the sacsin gene. Unfortunately, the genetic test is difficult to obtain, among others, because the size of the SACS gene is very large, and therefore its testing is expensive and time consuming.

Congenital malformations of the spinal cord usually appear in childhood, but their first symptoms can also occur in adulthood. Symptoms are not specific and can include back pain, gait disturbance, muscular atrophy, spasticity, and urologic complaints. Congenital cord malformations usually occur in the lower thoracic or upper lumbar area, although they have been reported in other locations.

There are still many misunderstandings about the pathological definitions and clinical significance of spinal cord defects. Traditionally, three main forms of malformations of the human spinal cord are reported: 1) dimyelia - a complete duplication of the spinal cord, roots and meninges (twin spinal cords), 2) diplomyelia - in which an accessory spinal cord with its own leptomeningeal but a common dural sac is present, and 3) diastematomyelia - a partial or complete sagittal clefting of the spinal cord into two hemicords (not necessarily symmetrical) with common meninges but with their own central canal and spinal roots [9]. Often these terms were used interchangeably, which caused confusion. Therefore, based on detailed radiographic and surgical results, Dias and Pang proposed a new unifying classification [7]. The new classification was based on the hypothesis that all spinal cord defects are caused by an embryo defect at the gastrulation stage [8], and recommended the term split cord malformations (SCM) for all types of double spinal cord malformations. According to this proposal, the spinal cord malformations are divided into two types: type I SCM consists of two hemicords, each contained within its own dural tube and separated by a dura-sheathed rigid 


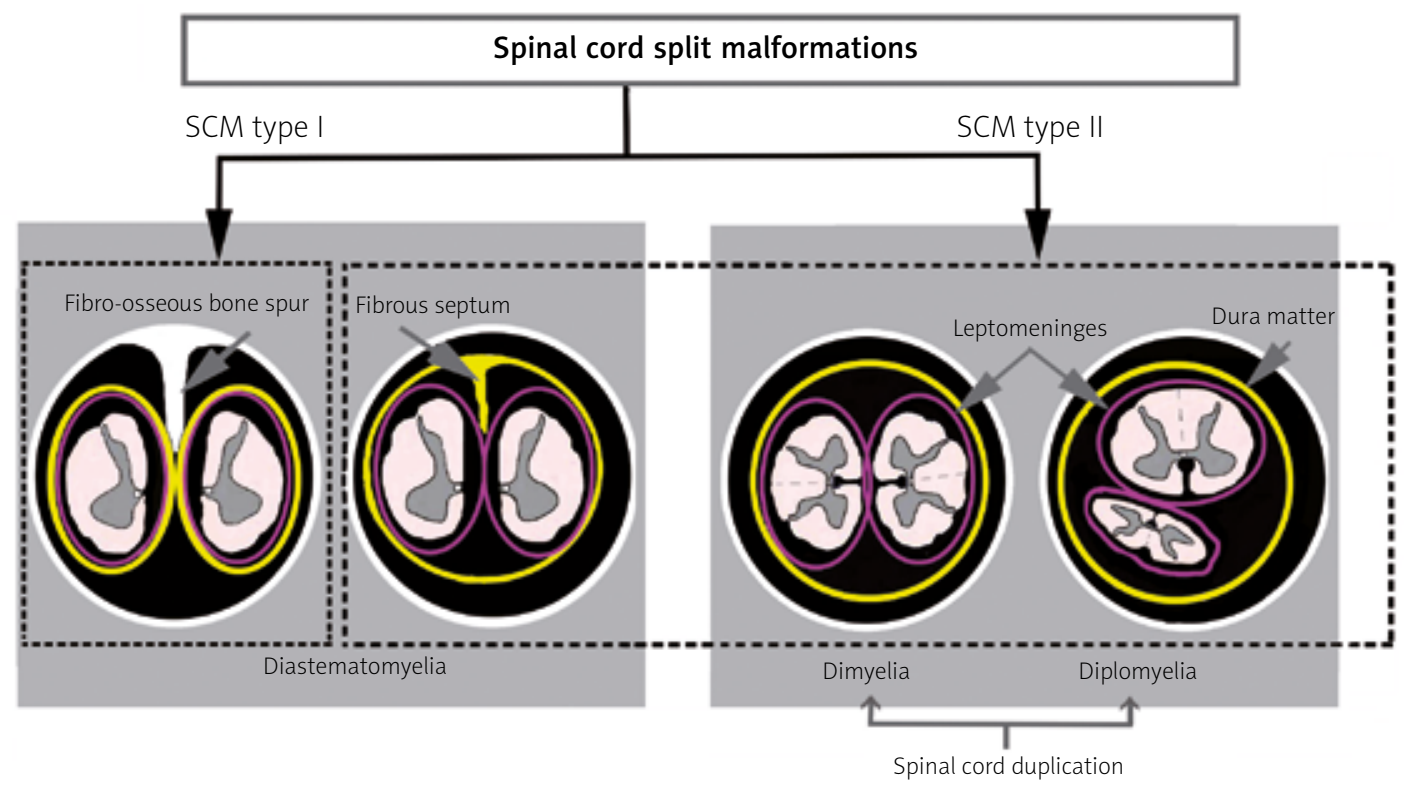

Fig. 5. Former and new classifications of the spinal cord malformations.

osseocartilaginous median septum, and type II SCM consists of two hemicords housed in a single dural tube separated by a fibrous median septum. However, introducing a new classification did not explain all doubts and much confusion still exists concerning the pathological definitions and clinical significance of double spinal cord malformations. Some of the authors identify SCM type I with diastematomyelia and SCM type II with diplomyelia [10]. The term "hemicord" originally used by Dias and Pang, may suggest the presence of two halves of the spinal cord, not its true duplication, and according to some authors, only the term "diastematomyelia" should be replaced by a more universal term "split cord malformation" [6]. Therefore, despite Dias and Pang's classification, the traditional terms for dysraphic malformations are still used because of the clarity of their names. The term "diastematomyelia" originates from the Greek words "diastema" (cleft) and "myelos" (marrow or medulla) and is used to describe a developmental malformation characterized by a splitting of the cord into at least two independent segments. In turn, diplomyelia and dimyelia are two forms of the spinal cord duplications. Dimyelia expresses the condition of complete duplication of the spinal cord, analogously to the term "dicephalus" describing a foetus or a person with two heads, and using of the term "dimyelia" should be limited to cases of an isolated, properly developed additional spinal cord [9]. Diplomyelia is a form of dimyelia in which the additional spinal cord exhibits disturbed size or internal structures (Fig. 5).

In diplomyelia, there is no clear correlation between clinical symptoms and the extent of spinal cord malformation. Some patients show minimal impairment, such as the absence of deep reflexes while others may show signs of increasing neurologic deterioration. Symptomatic cases often present with progressive motor and sensory symptoms. Back and leg pain is often seen in this condition, particularly in adults. Bladder and bowel symptoms are also common, although sometimes signs of neurogenic bladder may only be detected after urodynamic testing. Diplomyelia occurs usually in the spinal areas from the midthoracic to the low lumbar segments. In our case the location of the defect at C4-C5 levels was atypical. Moreover, our patient demonstrated symmetrical lower paraparesis, what is also atypical because the side of the hypoplastic cord is usually consistent with the lateralization of the neurologic symptoms or signs of the "orthopaedic syndrome" such as scoliosis or foot deformity [5].

Although our patient underwent two MRI scans of the spinal cord, diplomyelia was not found in vivo due to the lack of imaging studies that would also reveal a short two-segmental part of the spinal cord (C4-C6) located on the border region of both scans. Unfortunately, split cord malformations can be missed by routine imaging, especially if imaging was performed in the past using older technology. Since previous nor- 
mal results do not exclude the presence of the defect, it is now recommended for patients with suspected congenital spinal defects to visualize the entire neuroaxis using new neuroimaging methods. This should be remembered because early identification of spinal cord malformations and their surgical correction can improve neurologic symptoms, especially when it is done immediately after noticing the deterioration.

\section{Disclosure}

The author reports no conflict of interest.

\section{References}

1. Bereznyakowa O, Dupre N. Spastic ataxias. In: The cerebellum disorders and treatment. Manto M (ed.). TAGM Huisman, Elsevier, Amsterdam, Oxford, Cambridge 2018; 155: 191-195.

2. Bouhlal Y, Amouri R, E Euch-Fayeche G, Hentati F. Autosomal recessive spastic ataxia of Charlevoix-Saguenay: an overview. Parkinsonism Relat Disord 2011; 17: 418-422.

3. Bouchard JP, Barbeau A, Bouchard R, Bouchard RW. Autosomal recessive spastic ataxia of Charlevoix-Saguenay. Can J Neurol Sci 1978; 5: 61-69.

4. Bouchard JP. Recessive spastic ataxia of Charlevoix-Saguenay. In: Hereditary neuropathies and spinocerebellar atrophies. Handbook of clinical neurology. Vinken PJ, Bruyn GW, Klawans HL, de Jong JMBV (eds.). Elsevier, Amsterdam 1991; 16: 451-459.

5. Bret P, Patet ID, Lapras C. Diastematomyelia and diplomyelia. In: The Pediatric Spine II. Raimondi AJ, Choux M, Di Rocco C (eds.). Springer-Verlag, New York Inc. 1989; 91-112.

6. Dewan MC, Wellons JC. Spinal dysraphism and tethered spinal cord. In: Principles of neurological surgery. Ellenbogen RG, Sekhar LN, Kitchen N (eds.). Elsevier, Philadelphia 2018; 116-132.

7. Dias MS, Pang D. Split cord malformations. Neurosurg Clin N Am 1995; 6: 339-358.

8. Dias MS, Walker ML. The embryogenesis of complex dysraphic malformations: a disorder of gastrulation? Pediatr Neurosurg 1992; 18: 229-253.

9. Hori A, Fischer G, Dietrich-Schott B, Ikeda K. Dimyelia, diplomyelia, and diastematomyelia. Clin Neuropathol 1982; 1: 23-30.

10. Nelson SL, Johnston M. Split cord malformation updated. www. medlink.com index.php/article/split_cord_malformation, 2018.

11. Parfitt DA, Michael GJ, Vermeulen E, Prodromou NV, Webb TR, Gallo JM, Cheetham ME, Nicoll WS, Blatch GL, Chapple JP. The ataxia protein sacsin is a functional co-chaperone that protects against polyglutamine-expanded ataxin-1. Hum Mol Genet 2009; 18: 1556-1565.

12. Synofzik M, Soehn AS, Gburek-Augustat J, Schicks J, Karle KN, Schüle R, Haack TB, Schöning M, Biskup S, Rudnik-Schöneborn S, Senderek J, Hoffmann KT, MacLeod P, Schwarz J, Bender B, Krüger S, Kreuz F, Bauer P, Schöls L. Autosomal recessive spastic ataxia of Charlevoix Saguenay (ARSACS): expanding the genetic, clinical and imaging spectrum. Orphanet J Rare Dis 2013; 8: 41. 